\title{
Diferenças sazonais de quedas e fraturas em idosos gaúchos
}

\author{
Seasonal differences in falls and fractures among the elderly \\ in the southern Brazilian state of Rio Grande do Sul
}

Iride Cristofoli Caberlon ${ }^{1}$

Ângelo José Gonçalves Bós ${ }^{2}$

${ }^{1}$ Centro de Ciências da Saúde, Universidade Luterana do Brasil. Av. Itacolomi 3.600, São

Vicente. 94170-240

Gravataí RS Brasil.

iridec27@gmail.com

${ }^{2}$ Instituto de Geriatria e

Gerontologia, Pontifícia

Universidade Católica do

Rio Grande do Sul.

\begin{abstract}
Falls and fractures in the elderly represent a significant public health problem, associated with higher rates of morbidity and mortality, reduction of functional capacity, institutionalization of the elderly and early death. The scope of this study was to investigate falls and fractures in the elderly living in the metropolitan and mountainous region of the state of Rio Grande do Sul, analyzing the associated factors, seasonality and severity thereof. It is a cross-sectional, retrospective, descriptive, analytical and quantitative study of the elderly attended for falls from January 1 to December 31 of 2010 using secondary data from emergency units of the Brazilian Unified Health System. Data were collected from all health care reports filed in medical departments. The total number of bulletins for the elderly attended for falls was 6,556: $71 \%$ were women; $26.8 \%$ were in winter; $30 \%$ involved fractures (32\% women and $28 \%$ men $)(p<0.0001)$. In only $17.2 \%$ of the cases the fall site was recorded, and $58 \%$ were outside the home. Winter was the season with $34 \%$ of confirmed fractures $(p=0.0002)$ with more severe outcomes (26.3\%). Most falls and their consequences can be prevented and avoided. It is paramount to implement programs and multifactorial actions for intervention.
\end{abstract}

Key words Falls, Bone fractures, Associated risk factors, Seasonality, Fall severity, Health of the elderly
Resumo Quedas e fraturas em pessoas idosas representam um problema relevante de saúde pública. Ambas vêm associadas a elevados indices de morbimortalidade, redução da capacidade funcional, institucionalização do idoso e óbito precoce. O objetivo deste estudo foi investigar quedas e fraturas em idosos, residentes em municípios da região metropolitana e serra gaúcha do Rio Grande do Sul, analisando fatores associados, sazonalidade e gravidade. Trata-se de estudo transversal, retrospectivo, descritivo-analítico, quantitativo, com dados secundários, em idosos atendidos por queda entre primeiro de janeiro a 31 de dezembro de 2010, em unidades de Atendimento de Urgência e Emergência do SUS. A amostra foi de 6.556 idosos atendidos por queda, dos quais $71 \%$ eram mulheres, $26,8 \%$ dos atendimentos ocorreram no inverno, 30\% dos que caíram fraturaram, sendo $32 \%$ em mulheres contra $28 \%$ em homens ( $p<$ 0,0001 ). O local da queda foi registrado somente em $17,2 \%$ dos boletins, sendo $58 \%$ fora do domicílio. O inverno foi a estação do ano com $34 \%$ de fraturas confirmadas ( $p=0,0002)$, sendo $26,3 \%$ com gravidade severa. Como a maioria das quedas e suas consequências podem ser prevenidas e evitadas, urge criar programas e ações multifatoriais de intervenção.

Palavras-chave Quedas, Fraturas ósseas, Fatores de risco associados, Sazonalidade, Gravidade da queda, Saúde do idoso 


\section{Introdução}

O processo de envelhecimento traz consigo, simultaneamente, aumento dos fatores de risco e de comorbidades, especialmente como as doenças crônico-degenerativas ${ }^{1}$. No Brasil, um terço dos indivíduos que chegam aos 70 anos apresenta doenças crônicas degenerativas não transmissíveis e pelo menos $20 \%$ desses idosos terá algum grau de incapacidade associada, implicando diminuição da capacidade física e restrições à autonomia e à independência ${ }^{2}$.

A idade avançada e as mudanças provocadas pelas morbidades associadas afetam os múltiplos sistemas do organismo que controlam o equilíbrio, a locomoção, a mobilidade e a marcha, aumentando assim o risco de quedas nas pessoas idosas ${ }^{3}$. As quedas são um importante fator causal para a dependência dos idosos, pois, estão relacionadas a um índice elevado de incapacidade funcional e de morbimortalidade ${ }^{3}$.

Segundo Paixão Júnior e Heckman ${ }^{4}$, a queda só ocorrerá se estiverem presentes, duas condições: perturbação do equilíbrio e falência por parte do sistema de controle postural, em compensar essa perturbação $0^{4}$. Quanto mais frágil o paciente, mais suscetível a quedas e apresentando maior gravidade e maior probabilidade de fratura ${ }^{4}$.

As quedas ocorrem como resultado de uma complexa interação de fatores de risco que podem ser classificados em biológicos, como idade, gênero e raça, associados às mudanças devidas ao envelhecimento; em fatores de risco comportamentais, os que dizem respeito às ações humanas, emoções ou escolhas diárias e são potencialmente modificáveis; os fatores de risco ambientais que incluem a interação das condições físicas dos indivíduos e as do ambiente que os cerca; e os fatores de riscos socioeconômicos, como desigualdades de trabalho/renda, educação, habitações sem condições de saneamento básico, acesso limitado ao cuidado de saúde e assistência social em áreas prioritárias e deficiência de recursos da comunidade $^{5-7}$. O dano mais comum de uma queda é a fratura. Nos idosos, o trauma mais prevalente é o de fêmur, seguido pelo trauma de radio e de clavícula $^{8}$. O medo de cair novamente é outra consequência grave, pois pode levar o idoso a diminuir ou restringir suas atividades da vida diária. Assim, o idoso terá dificuldade em realizar estas atividades cotidianas, e, com a restrição, pode desenvolver diminuição da força muscular, levando ao enfraquecimento geral, dependência física e psicológica, isolamento social e possível institucionalização ${ }^{8}$.
O Rio Grande do Sul é um dos estados brasileiros com maior distinção entre as quatro estações do ano, onde ocorrem nestas grandes diferenças sazonais, com significativas oscilações relacionadas ao clima, temperatura, e umidade nas suas diferentes regiões, os quais representam fatores ambientais que interferem na saúde do indivíduo. Estes fatores sazonais podem ocasionar problemas leves à graves nos sistemas respiratórios, cardiovasculares e osteomusculares, principalmente em idosos ${ }^{2}$. Entretanto, não encontramos na literatura pesquisada trabalhos que avaliassem a importância da variação sazonal sobre a incidência de quedas e fraturas em idosos.

Com base nesta fundamentação científica, investigou-se que fatores, particularmente as estações do ano (sazonalidade), estariam associados na avaliação de quedas e fraturas em idosos (60 anos e mais) que residem em municípios da região metropolitana e da serra gaúcha do Estado do Rio Grande do Sul/Brasil.

\section{Metodologia}

Foram identificados todos os idosos (60 anos e mais) atendidos por queda no ano de 2010, em quatro unidades de Atendimento de Urgência e Emergência Hospitalar (UAUEH) do Sistema Único de Saúde (SUS) do Estado do Rio Grande do Sul (RS). Foram escolhidas duas UAUEH no interior do Estado (uma na Região Metropolitana e outra na Região Serrana) do Rio Grande do Sul e as duas UAUEH da capital do Estado, Porto Alegre. Mediante revisão dos boletins de atendimento, arquivados no Sistema de Registro Médico de cada hospital pesquisado, foram extraídos dados referentes ao idoso, data e horário do atendimento, data e horário da queda, local da queda, realização ou não de exame radiográfico, sinais vitais e consequências da queda.

Inicialmente, buscaram-se observar as possíveis relações nas distribuições das frequências dos diferentes sexos (masculino e feminino) e a faixa etária (em década de idade) dos idosos atendidos e o resultado do estudo radiológico realizado ou não durante o atendimento. A investigação radiográfica foi caracterizada em quatro níveis: fratura confirmada, sem fraturas, radiografia não realizada e sem informação. A frequência dos resultados da investigação radiográfica foi observada em relação à frequência de quedas em cada estação do ano. O número de fraturas registradas foi tratado como variável numérica e a média calculada para cada estação do ano (verão, 
outono, inverno e primavera). Visto não existir na literatura nenhuma escala de gravidade das quedas, as informações sobre fratura, presença de escoriação, edema, equimose e dor foram classificadas criando uma classificação das consequências da queda conforme a gravidade das lesões apresentadas. Diferentes lesões receberam notas diversas conforme a gravidade atribuída. As fraturas receberam nota 10 , escoriações 5 , equimose 4 e edema e dor 1 . Desta forma uma pessoa que teve uma fratura (10), uma escoriação (5) e uma equimose (4) receberam gravidade 19. Os níveis de gravidade foram posteriormente classificados conforme a distribuição observada em zero (sem gravidade), 1 (gravidade muito leve), 2 a 4 (leve), 5 a 9 (moderada), 10 a 14 (severa) e 15 ou mais (muito severa).

A distribuição dos idosos conforme o grau de gravidade da queda foi calculada para cada estação do ano. As tabelas foram testadas pelo qui-quadrado e as diferenças entre as médias pela análise de variância - ANOVA. A probabilidade de fratura (risco) foi calculada através da regressão logística para as variáveis estações do ano, idade e gênero. Foi considerado estatisticamente significativo um teste com o p $<0,05$ e indicativo de significância um $\mathrm{p}<0,1^{9}$. Foi utilizado o software EPI INFO for Windows 3.5.1. O estudo foi aprovado pelo Comitê de Ética em Pesquisa da PUCRS, sob o protocolo 04881/2009 e pelas instituições hospitalares sob os protocolos 562/2011, 049/2011, 036/2011 e SMS 2010. Os autores declaram não haver conflito de interesses no presente trabalho.

\section{Resultados}

No período de $1^{\circ}$ de janeiro a 31 de dezembro de 2010, foram atendidas 6633 pessoas com 60 anos e mais, pelo motivo queda, nas quatro unidades de UAUEH estudadas. As quatro unidades estudadas e uma unidade representativa da Região da Serra Gaúcha, com a cobertura de 29,5\% dos 133 mil idosos residentes na Região, no ano estudado; e três unidades representativas da Região Metropolitana, com a cobertura de 80,3\% dos 297.599 idosos residentes na Região, no ano estudado. Foram excluídas do estudo 1,2\% do total, sendo $24(0,36 \%)$ por sexo não informado, $19(0,29 \%)$ por idade menor de 60 anos ou sem idade, e $34(0,51 \%)$ por residirem em municípios de outra região do estudo. Entre os excluídos, 20 tiveram fratura confirmada pela radiografia, 44 fizeram radiografia sem fratura e 12 não realiza- ram a investigação radiográfica. Assim, o total da população que preencheu os critérios de inclusão do estudo, ficou em 6.556 idosos (98,8\%) com 60 anos e mais, como demonstra a Tabela 1 . O número amostral apresenta um poder estatístico de $74,24 \%$ para as variáveis: queda e fratura entre as estações do ano.

A idade dos sujeitos estudados variou de 60 anos a 104 anos de idade, dos quais $71,1 \%$ eram mulheres e 28,9\% homens. Em relação à faixa etária dos idosos atendidos, 42,5\% tinham entre 60-70 anos de idade e deste percentual, 33,1\% eram do sexo masculino. Somente 3,2\% tinham 90 anos e mais, sendo que $84,7 \%$ destes eram do sexo feminino. Quanto maior a faixa etária, maior foi o percentual do sexo feminino e menor o percentual do sexo masculino. Foram atendidas 10 pessoas com 100 anos e mais.

O período de atendimento em relação à estação do ano foi significativo $(\mathrm{p}=0,0021)$. O maior percentual incidiu nos atendimentos realizados na estação do inverno à tarde (29,1\%), vindo em segundo lugar a estação do verão com $27,1 \%$ na madrugada, $26,1 \%$ na noite, e $25,9 \%$ na manhã, respectivamente.

Quanto à variável local da queda, verificou-se uma frequência de 82,8\% (5.427) dos casos em que o local da queda não foi relatado, por isso não foram descritos. O sinal vital mais frequentemente relatado foi o da pressão arterial cuja frequência ficou entre $32 \%$ no inverno e $28,9 \%$ no verão. A Frequência Cardíaca foi verificada e registrada entre $19,7 \%$ no inverno e $15,8 \%$ no verão. A saturação de Oxigênio (SAT) foi medida entre $11,5 \%$ dos atendimentos no inverno, e $6,5 \%$ na primavera.

Verificou-se, que 92,1\% (5991) dos idosos que caíram realizaram Investigação Radiográfica (IR), 7,9\% (517) não a realizaram e 0,7\%(48) sem informação. Do total de idosos que realizaram a IR, 30,6\% (2008) tiveram fratura confirmada e, destes, 31,9\% eram do sexo feminino, ficando demonstrado que este percentual foi maior nas mulheres que nos homens $(28,2 \%)$, quando $\mathrm{p}<0,0001$. Com relação aos idosos atendidos por queda após a IR e a consequência da queda e a estação do ano (Tabela 2), verificou-se que a estação do inverno foi a que teve a maior percentagem, 33,9\% (596) de fraturas confirmadas e a primavera com o menor percentual, $27,3 \%$ (436), com $\mathrm{p}=0,0002$.

A comparação das médias do número de fraturas pela estação do ano (Tabela 3 ) demonstrou que a estação do inverno foi a que apresentou mais fraturas com média de 1,13 fraturas por 
pessoa, vindo em segundo lugar a estação do verão com média de 1,12 , com $\mathrm{p}=0,025$.

$\mathrm{Na}$ Tabela 4, obteve-se que $15,5 \%$ das pessoas idosas tiveram queda sem gravidade. Verificou-se que dentre as estações do ano a que apresentou maiores níveis de gravidade, ou seja, muito severa, severa e moderada foi a estação do inverno, respectivamente com os percentuais de $28,6 \%$, $28,4 \% 28,3$. Nota-se que as estações do inverno e outono foram as que apresentaram maiores níveis de gravidade de queda, $(\mathrm{p}=0,0011)$.

O resultado da regressão logística, calculando a chance de fratura entre o verão e as outras estações do ano, demonstrou que as chances de fratura foram significativamente maiores nas quedas ocorridas no inverno e outono. Ou seja, quedas no inverno têm 23\% mais chance de sofrerem fratura que no verão, essa associação com o outono foi menor (17\%). Essa associação manteve-se significativa mesmo ajustando para o sexo e a idade do atendido. Idade e sexo feminino foram fatores significativos para o risco de queda (Tabela 5).

No modelo univariado, as mulheres têm 19,4\% maior chance de cair e fraturar do que os homens que caem independente da estação do ano. Ajustando por idade, a chance das mulheres caírem diminui para 15\%, continuando signifi-

Tabela 1. Distribuição dos idosos atendidos por queda segundo sexo do atendido por faixa etária e resultado radiográfico. Ano de 2010, RS/Brasil.

\begin{tabular}{|c|c|c|c|c|}
\hline & Feminino & Masculino & Total & p \\
\hline Faixa etária (anos) & & & & $<0,001$ \\
\hline $60-<70$ & $1867(66,9 \%)$ & $923(33,1 \%)$ & $2790(42,5 \%)$ & \\
\hline $70-<80$ & $1751(73,0 \%)$ & $648(27,0 \%)$ & $2399(36,6 \%)$ & \\
\hline $80-<90$ & $869(75,0 \%)$ & $289(25,0 \%)$ & $1158(17,7 \%)$ & \\
\hline 90 e mais & $177(84,7 \%)$ & $32(15,3 \%)$ & $209(3,2 \%)$ & \\
\hline Resultado radiográfico & & & & $<0,001$ \\
\hline Fratura confirmada & $1480(31,7 \%)$ & $528(27.9 \%)$ & $2008(30,6 \%)$ & \\
\hline Sem fratura & $2829(60.7 \%)$ & $1154(61,0 \%)$ & $3983(60.8 \%)$ & \\
\hline RX não realizado & $324(6.9 \%)$ & $193(10,2 \%)$ & $517(7,9 \%)$ & \\
\hline Sem Informação & $31(0.7 \%)$ & $17(0.95)$ & $48(0.7 \%)$ & \\
\hline Total & $4664(71,1 \%)$ & $1892(28,9 \%)$ & $6556(100,0 \%)$ & \\
\hline
\end{tabular}

Fonte: Formulário do estudo.

Tabela 2. Distribuição dos idosos atendidos por quedas segundo a estação do ano e os resultados da investigação de fraturas. Ano de 2010, RS/Brasil.

\begin{tabular}{lrrrrr}
\hline \multicolumn{1}{c}{ Estação do ano } & Inverno(\%) & Outono (\%) & Primavera(\%) & Verão(\%) & Total (\%) \\
\hline Fratura confirmada & $596(33,9)$ & $515(32,1)$ & $436(27,3)$ & $461(28,9)$ & $2008(30,6)$ \\
Sem fratura & $1023(58,2)$ & $961(59,8)$ & $1017(63,8)$ & $982(61,5)$ & $3983(60,8)$ \\
RX não realizado & $124(7.1)$ & $115(7,2)$ & $128(8,0)$ & $150(9,4)$ & $517(7,9)$ \\
Sem informação & $15(0,09)$ & $15(0.09)$ & $14(0,9)$ & $4(0,03)$ & $48(0.7)$ \\
Total & $1758(26,8)$ & $1606(24,5)$ & $1595(24,3 \%)$ & $1597(24,4)$ & $6556(100,0 \%)$ \\
\hline
\end{tabular}

$\mathrm{p}=0,0002$

Tabela 3. Média do número de fraturas para cada a estação do ano. Ano de 2010, RS/Brasil.

\begin{tabular}{lccccc}
\hline Número de fraturas & Inverno(\%) & Outono (\%) & Primavera(\%) & Verão(\%) & \\
\hline Média (n) & 1,1301 & 1,0594 & 1,0365 & 1,1266 & $\mathrm{p}=0,025$ \\
Desvio Padrão & 0,8319 & 0,2598 & 0,2214 & 0,7918 & \\
Máximo & 17 & 4 & 4 & 11 & \\
\hline
\end{tabular}


Tabela 5. Resultado da análise do risco de fratura atribuída a outras estações do ano comparadas com o verão, ajustado para sexo e idade do idoso atendido por queda. Ano de 2010, RS/Brasil.

\begin{tabular}{|c|c|c|c|}
\hline & RC & $95 \%$ IC & p \\
\hline \multicolumn{4}{|l|}{ Estação do ano } \\
\hline $\begin{array}{l}\text { Verão } \\
\text { (referência) }\end{array}$ & 1 & & \\
\hline Inverno & 1.2335 & $1.0648-1.4288$ & 0.0051 \\
\hline Outono & 1.1734 & $1.0092-1.3644$ & 0.0377 \\
\hline Primavera & 0.9177 & $0.7862-1.0712$ & 0.2767 \\
\hline \multicolumn{4}{|l|}{ Sexo } \\
\hline $\begin{array}{l}\text { Masculino } \\
\text { (referência) }\end{array}$ & 1 & & \\
\hline Feminino & 1,1500 & $1,0208-1,2956$ & 0,0216 \\
\hline $\begin{array}{l}\text { Idade do atendido } \\
\text { (anos) }\end{array}$ & 1,0217 & $1,0155-1,0280$ & $<0,0001$ \\
\hline
\end{tabular}

RC= Razão de Chance; $\mathrm{IC}=$ Intervalo de Confiança.

cativa a diferença entre homens e mulheres. $\mathrm{Na}$ análise univariada, cada ano a mais de vida corresponde ao risco calculado de $2,57 \%$ maior de fratura. A idade, no modelo ajustado para sexo e estação do ano é significativa. Uma pessoa um ano mais velha tem $2,2 \%$ mais chances de fraturar ao cair. Não ocorreu interação significativa entre idade e sexo em relação à fratura, apesar da idade dos homens que fraturaram ter sido significativamente maior dos que não fraturaram. $\mathrm{O}$ local de atendimento não influenciou as chances das pessoas idosas caírem.

\section{Discussão}

A descrição da frequência de quedas e de fraturas numa população de idosos é bastante variada e depende de diferentes aspectos individuais da mesma, tais como idade, sexo, hábitos e estilo de vida, características clínicas individuais e das ambientais do local da queda. Todos os anos, um em cada três idosos cai, podendo este evento levar à lesão, hospitalização, perda da independência e até a morte ${ }^{2,8}$.

O presente estudo avaliou 6556 idosos que foram atendidos em quatro UAUEH, localizadas na Região Metropolitana e na Serra Gaúcha do RS/Brasil, em 2010. Encontrou-se como resultado em relação a variável idade dos idosos estudados: variação de 60 a 104 anos de idade, sendo que, dentro desses limites, encontrou-se 10 pessoas com 100 anos e mais. Entre os idosos observados, $71,1 \%$ eram mulheres e $28,9 \%$ homens. Se considerarmos que $30 \%$ de idosos caem a cada ano, estima-se que 60.000 idosos teriam caído num ano. Contabilizando os 5.749 idosos atendidos, somente em Porto Alegre, teríamos uma proporção menor de $10 \%$ dos idosos que caem atendidos. Em relação à faixa etária dos idosos atendidos, apesar do grupo entre 60-69 anos de idade apresentar os maiores percentuais $(42,5 \%)$, os mesmos ficaram abaixo do percentual esperado para a faixa etária, pois, de acordo com o último censo do $\mathrm{IBGE}^{10}, 55,4 \%$ dos idosos gaúchos estão na referida faixa etária. O percentual de idosos que caíram nessa faixa etária (entre 60 e 69) corresponde a $23 \%$ menos que o observado na população. Essa observação é válida tanto para homens quanto para mulheres. As demais faixas etárias apresentam percentuais mais elevados que o encontrado no último censo. A diferença percentual entre as faixas etárias dos idosos que caíram e o observado no censo, aumenta conforme o avançar das mesmas. Quanto maior a faixa etária, menor foi o percentual do sexo masculino. Segundo a revisão da literatura, diferentes estudos científicos realizados por vários 
autores referem que cerca de $30 \%$ das pessoas idosas caem a cada ano e confirmam a observação da presente pesquisa, que idosos com maior idade caem mais ${ }^{2,11,12}$. Os idosos de 75 a 84 anos que necessitam de ajuda nas atividades da vida diária têm maior probabilidade de cair com um percentual de quatorze vezes mais do que pessoas da mesma idade que são independentes ${ }^{13}$.

Observamos que as quedas foram mais frequentes e graves nas mulheres. Pode-se enumerar como fatores relacionados à queda em mulheres a menor massa e força muscular, viuvez e uso comum de psicotrópicos ${ }^{14}$. E idosos ativos tendem a cair mais em ambientes externos ${ }^{15}$. Talvez os idosos homens tendam a se expor menos que as mulheres que se mantêm mais ativas, tanto no lar quanto socialmente. Um fato que corrobora esta hipótese é a observação de que entre os 173 atendidos com relato de terem caído dentro do ônibus, 139 (80,3\%) eram mulheres.

Demonstrou-se que há relação entre queda e estação do ano (sazonalidade da queda), sendo o inverno a estação com maior número de quedas. $\mathrm{O}$ inverno foi a estação que teve o maior percentual de fraturas confirmadas, seguida do outono, que teve a maior incidência de quedas à noite. Estudos realizados afirmam que a iluminação inadequada é um fator de risco extrínseco importante ${ }^{14}$. O presente estudo demonstrou que há relação entre a estação do inverno e quedas, fraturas e sua gravidade. Estes resultados vão ao encontro do que afirmaram os autores referendados $^{6,16-18}$ na revisão da literatura. Nos meses mais frios do ano a locomoção nos idosos fica mais comprometida, os reflexos ficam lentos para reagir e se proteger dos riscos, aumenta a necessidade de uso de vestuário mais adequado, do contrário o corpo é incapaz de compensar a perda do calor e a diminuição de sua temperatura interna ${ }^{17,18}$.

Assim, a partir de maio até agosto, quando as temperaturas no Rio Grande do Sul caem próximas a zero, provocando alteração nos fatores climáticos (frio e umidades intensas, baixas temperaturas, velocidade acelerada do vento, geadas, neve e chuvas), podem aumentar a frequência de quedas e fraturas. Na estação do inverno, quando o frio é rigoroso e úmido, os idosos suam menos, ingerem mais líquidos quentes e, por consequência, têm necessidade de ir mais vezes ao banheiro, durante a noite, ficando mais expostos ao risco de quedas $^{6,17,18}$. Por isso é importante avaliar o risco e a gravidade da exposição ao frio, levando-se em consideração a temperatura do ar, a velocidade do vento e a atividade física, sem esquecer os as- pectos nutricionais (desidratação e desnutrição e necessidades de vitamina $\mathrm{D}$ e cálcio $)^{6,18}$.

Um estudo com idosos que caíram em Uberlândia, MG, encontrou resultado contrastante, com maior frequência de quedas no verão (período de chuvas) e na parte da manhã ${ }^{14}$. O estudo de Uberlândia não procurou observar a severidade das quedas, mas demonstrou que as condições climáticas do verão na cidade apresentam características semelhantes ao inverno na região metropolitana de Porto Alegre e Serra Gaúcha, onde também ocorre forte período de chuvas.

A queda, síndrome multifatorial e heterogênea, pode ser considerada um dos principais eventos incapacitantes para os idosos, devido à interação complexa que existe entre perdas individuais (fator intrínseco), perigos ambientais (fatores extrínsecos) e aspectos relacionados à realização da ação (fator situacional) ${ }^{19}$. Está comprovado que um terço dos idosos da comunidade cai ao menos uma vez ao ano. Apesar de sua importância, durante as consultas médicas, os episódios de quedas são pouco abordados.

As quedas apresentam forte relação com o envelhecimento principalmente pela alta prevalência de instabilidade postural, declínio da acuidade visual, perda da audição, distúrbios do equilíbrio, marcha e coordenação motora, sendo assim mais suscetíveis aos perigos ambientais ${ }^{20}$.

Pesquisas sugerem que as chances de quedas aumentam significativamente com o avançar da idade e que os idosos jovens, na maioria das vezes, sofrem quedas menos sérias ou se recuperam mais facilmente das consequências das mesmas e assim podem reportar menos os episódios de quedas ${ }^{4}$. Em um estudo realizado entre os idosos que caíram $48 \%$ apresentavam faixa etária entre $80-89$ anos e $66 \%$ eram do sexo feminino ${ }^{20}$. Entre os idosos que vivem em comunidade, as quedas são mais comuns nas mulheres, especialmente aquelas com osteoporose no período pós menopausa $^{3}$.

Um estudo realizado em Oslo, Noruega, também constatou que ocorre elevada incidência e gravidade de quedas no inverno, o que foi atribuído às precipitações e ao acúmulo de neve ${ }^{21}$. Essa explicação não se aplica a nossa realidade, pois não houve relato de queda de neve nos locais pesquisados, no ano de 2010. No Rio Grande do Sul há formação de geadas que ocorrem no período da madrugada nos meses do inverno, provocando um frio intenso e úmido, com temperaturas próximas a zero ou inferior a este. Analisando os resultados dos 1546 atendimentos com relato de horário da queda, grande parte dos mesmos 
ocorreu pela manhã com predomínio na estação do verão. O predomínio também foi maior no inverno para os que caíram de tarde. Além da falta de informação sobre o horário da queda, observou-se também falta de informações importantes no relato de uma queda. O local da queda foi relatado em menos de $20 \%$ dos atendimentos, sendo o mais frequente, queda dos idosos fora do domicílio. Um estudo comparativo das características do trauma entre 2075 pacientes, incluindo idosos, realizado em São Paulo, apontou que estes foram mais frequentemente vítimas de queda da própria altura (41\%) e de atropelamento $(28,6 \%)$, enquanto no grupo não idoso predominaram, significativamente, os acidentes com motocicletas $(28 \%)^{22}$. O idoso cai frequentemente no seu domicílio, o que pode acarretar muitas sequelas ou até mesmo a morte, dependendo da gravidade $^{23}$. Estudo realizado em 2007 mostrou que a maior parte das quedas ocorreu após deslizes em piso molhado e que as participantes possuem conhecimentos sobre os fatores causadores de quedas, mas estes obstáculos ainda persistem em seu ambiente ${ }^{23}$.

Acredita-se que os fatores que desencadeiam estes incidentes podem ser eliminados do ambiente domiciliar, através da adaptação o ambiente e mudanças de hábitos do idoso e da família $^{24}$. No estudo realizado em Buenos Aires, em 2009 , sobre os locais das quedas de idosos, os autores observaram que as quedas ocorreram mais no período da manhã, em local de piso áspero e seco, sem degraus, rampas ou tapetes, iluminação adequada e o tipo de calçado mais utilizado foi chinelo de borracha ${ }^{25}$.

As quedas, como muitas pessoas pensam, não são uma parte normal do envelhecimento. A maioria das quedas pode ser prevenida e evitada, assim como suas consequências. Estas são caracterizadas por lesões e fraturas. Elas podem gerar redução ou perda da autonomia, da independência, da qualidade de vida da pessoa idosa e aumento de danos sociais, financeiros, emocionais e mentais, levando à hospitalização, institucionalização e aumento da morbidade e mortalidade. A prevenção das quedas e fraturas faz-se mediante programas desenvolvidos por equipes de profissionais especializados na área de Geriatria e Gerontologia, nas unidades básicas e unidades de Saúde da Família e demais serviços de saúde do Sistema Único de Saúde. Essa atuação deve ter caráter multiprofissional na atenção integral e longitudinal ao idoso, numa ação conjunta com o cuidador familiar ou formal, visando à redução de seu risco e do medo de cair. A interven- ção busca incentivar a realização das atividades diárias e instrumentais do quotidiano sempre em locais seguros, tanto no domicílio como fora do mesmo, proporcionando assim qualidade de vida às pessoas idosas. Na longevidade é importante prevenir a fragilidade e resolver ou atenuar os problemas de saúde que estão na origem das quedas (DCDNT, causas externas, doenças neuropsiquiátricas e medicamentos de uso contínuo).

Existem vários relatos de experiências exitosas na implantação de programas de prevenção de queda em idosos. A maioria desses programas avalia o equilíbrio, a marcha, as medicações em uso e o ambiente de seu domicílio ${ }^{16}$. Estratégias de intervenção individualizada ou em pequenos grupos são eficazes e de baixo custo para a prevenção de quedas em idosos atendidos pelos serviços de atenção básica da saúde. A abordagem individualizada coaduna com a estratégia da Saúde da Família do SUS.

Verificou-se que os sinais vitais foram esparsamente registrados nos boletins de atendimento em todas as unidades pesquisadas, apesar dos sinais vitais fazerem parte dos critérios de prioridade no atendimento de urgência e emergência do Ministério da Saúde. A pressão arterial, item com maior frequência, foi verificada e registrada em menos de um terço dos boletins. Não se encontrou na literatura brasileira estudos sobre quedas e sua relação com os sinais vitais no momento do atendimento. O número reduzido de registros dos dados vitais dos pacientes idosos atendidos nas UAUEH é preocupante, não atendendo às diretrizes preconizadas pela Política de Atenção à Saúde do SUS preconizada pelo Ministério da Saúde (MS). A Portaria 2048 GM/ MS/2002 26 propõe a implantação, nas unidades de atendimento de urgências, do acolhimento e da "triagem classificatória de risco". Este processo "deve ser realizado por profissional de saúde, de nível superior, mediante treinamento específico e utilização de protocolos pré-estabelecidos e têm por objetivo avaliar o grau de urgência das queixas do paciente, colocando-os em ordem de prioridade para o atendimento".

Os resultados do estudo oportunizaram ainda identificar uma variável que não constava nos objetivos da investigação, o TCE. Na comparação do GC e o TCE, ficou demonstrado que, das 176 pessoas que tiveram TCE, mais da metade delas não foi verificado e/ou não foi medido e registrado o grau de consciência. Este percentual de registro do GC foi menor que das pessoas que não tiveram TCE, ficando os profissionais da saúde sem parâmetros clínicos iniciais do pa- 
ciente idoso para a tomada de decisão imediata mais adequada, a cada situação frente ao trauma sofrido. Ao longo do estudo, observou-se que, nas UAUEH, as equipes profissionais realizam o atendimento das vítimas de trauma, executando rotinas padronizadas de forma inadequada e/ou com certa negligência.

Pouca importância parece ter sido dada aos idosos que caíram no sentido de diagnosticar as possíveis causas e os fatores associados da queda. Essa constatação corrobora com os insipientes e pontuais programas existentes de prevenção de quedas em idosos no Brasil. Preocupa-nos também o fato dos profissionais de saúde não realizarem a verificação e/ou não executarem o registro sistemático dos sinais vitais no acolhimento idoso (vítima com trauma) na unidade de UAUEH. A não realização desta atividade impede e/ou exerce influência na tomada de decisão mais adequada dos cuidados no atendimento do usuário que se seguem. A conduta médica e de enfermagem precisa estar fundamentada em evidências, conforme as diretrizes do MS, e os parâmetros estabelecidos por protocolos e/ou consensos brasileiros na atenção às pessoas idosas e em geral. Esse cuidado é especificamente mais importante na atenção às pessoas idosas, nas UAUEH por necessitarem de uma avaliação mais ampla, levando sempre em conta outros riscos associados, as comorbidades, as DCDNT de que são portadores e o tratamento medicamentoso que estão utilizando (prescrito pelo seu médico). Na avaliação inicial, é fundamental que a equipe multiprofissional (enfermagem e médicos) verifique e registre os dados vitais, realize o acolhimento com a classificação de risco do paciente e execute as medidas de intervenção, monitorando o estado do paciente ao longo de todo o tempo em que é atendido na UAUEH. Por fim, é também necessário que se encaminhe o paciente ao seu médico ou a serviço especializado, para realizar a prevenção de quedas e o acompanhamento de seus problemas de saúde.

\section{Conclusões}

O presente trabalho confirmou as hipóteses alternativas levantadas quanto à sazonalidade da queda e sua gravidade. Neste estudo ficou demonstrado como resultados que existe relação significativa entre sexo e faixa etária dos idosos que participaram do estudo, pois, quanto maior a faixa etária, maior foi o percentual do sexo feminino. O período do dia do atendimento em relação à estação do ano foi significativo, quando o maior percentual incidiu nos atendimentos realizados na estação do inverno e à tarde. Quanto à gravidade da queda, o estudo mostrou que a estação do inverno apresentou maior nível de gravidade. Dessa forma, os idosos que caem no inverno têm mais fraturas e com maior gravidade que nas outras estações do ano.

Os autores concluem que, apesar dos esforços e ações implementadas na última década, pelos gestores responsáveis pela atenção integral aos idosos, quanto à execução de ações estratégicas de prevenção de quedas e fraturas, de um acolhimento e atendimento técnico humanizado adequado nas UAUEH, ainda na prática poucos avanços foram executados frente às demandas. Há ainda grandes desafios para serem superados quanto a sua efetivação, destacando-se: a capacitação e educação permanente das equipes multiprofissionais, quanto ao processo de envelhecimento e longevidade na rede de serviços de saúde, para a garantia da atenção integral; a criação de programas e ações de prevenção e de intervenção multifatoriais, de forma a atender às necessidades individualizadas e de grupos de idosos com necessidades similares; a reorganização dos serviços de atendimento nas UAUEH, com garantia de infraestrutura física adequada, material, equipamentos, tecnologia de informática e recursos humanos suficientes e qualificados, para assegurar a excelência da assistência nos serviços; implementação de linhas de cuidados aos idosos, rompendo os mitos culturais e de gênero na prevenção de quedas; promoção e educação do próprio idoso, cuidadores e familiares; além da adesão dos idosos às medidas de prevenção (mudança de comportamento) no autocuidado e suporte familiar e social no cuidado em seu domicílio e comunidade.

Essa reorganização é essencial também para a obtenção de dados completos e seguros sobre os atendimentos realizados, o monitoramento das ações para mensurar sua eficiência e eficácia, avaliando assim o impacto na qualidade de vida dos idosos. Os resultados da pesquisa apontam também para a necessidade de novos estudos epidemiológicos e clínicos que possam esclarecer outras questões relacionadas às abordagens sobre os fatores de risco de quedas e fraturas e programas multifatoriais de prevenção e de intervenção. 


\section{Colaboradores}

IC Caberlon participou da elaboração do projeto, execução da coleta e tabulação dos dados, acompanhou a análise dos dados e elaborou o manuscrito. AJG Bós orientou na elaboração do projeto, acompanhou a execução e tabulação dos dados, executou a análise dos dados e auxiliou na elaboração do manuscrito.

\section{Referências}

1. Siqueira FV, Facchini LA, Picchini RX, Tomasi E, Thumé E, Silveira DS, Vieira V, Hallal PC. Prevalência de quedas em idosos e fatores associados. Rev Saude Publica 2007; 41(5):749-756.

2. Brasil. Ministério da Saúde (MS). Secretaria de Atenção Básica. Departamento de Atenção Básica. Envelhecimento e saúde da pessoa idosa. Brasília: MS; 2007. (Série A. Normas e Manuais Técnicos).

3. Machado TR, Oliveira CJ, Costa FBC, Araujo LA. Avaliação da presença de risco para queda em idosos. Rev. Eletr. Enf. 2009; 11(1):32-38.

4. Paixão Júnior CM, Heckman MF. Distúrbios da postura, marcha e quedas. In: Freitas EV, Py L, Cançado F, Gorzoni M, Doll J, organizadores. Tratado de geriatria e gerontologia. $2^{\text {a }}$ ed. Rio de Janeiro: Guanabara-Koogan; 2006. p. 954-961.

5. Perracine MR. Prevenção e Manejo de Quedas no Idoso. 2006. [acessado 2013 maio 20]. Disponível em: http://www.pequi.incubadora.fapesp.br/portal/quedas.pdf

6. Center for Research and Prevention of Injuries CEREPRI. Fact sheet: Prevention of Falls among Elderly: Elderly Safety-Focus on Accidental Injuries. Atenas: Athens University; 2002.

7. Sociedade Brasileira de Geriatria e Gerontologia. Quedas em idosos: prevenção [monografia na internet]. Rio de Janeiro: Associação Medica Brasileira e Conselho Federal de Medicina; 2008 [acessado 2013 maio 31]. Disponível em: http://www.projetodiretrizes.org. br/projeto_diretrizes/082.pdf.

8. Jahana KO, Diogo MJDE. Quedas em idosos: principais causas e conseqüências. Saúde Coletiva 2007; 4(17):148-153.

9. Bós AJG. Epi Info sem mistérios: um manual prático. Porto Alegre: Edipucrs; 2004.

10. Instituto Brasileiro de Geografia e Estatística (IBGE). Censo 2010 [homepage]. [acessado 2013 maio 31]. Disponível em: http://www.ibge.gov.br/home/estatistica/ populacao/projecao_da_populacao/2008/projecao.pdf

11. Souza ACA. Quedas no Idoso. In: Terra NL, organizador. Entendendo as queixas do idoso. Porto Alegre: Edipucrs; 2003. p. 379-401.

12. Strumpf N, Wagner J, Evans L, Patterson J. Reducing restraints: Individual approaches to behavior. Huntingdon Valley: Geriatrc Research \& Training Center; 1992.

13. Carvalho J, Pinto J, Mota, J. Atividade Física, equilíbrio e medo de cair: um estudo em idosos institucionaliza-

14. dos. Rev. Port. Cien. Desp. 2007; 7(2):225-231.

Freitas-Junior OS. Queda de idosos que motiva atendimento hospitalar de emergência pelo SUS em Uberlândia - MG: epidemiologia e consequências para a saúde [dissertação]. Uberlândia: Universidade Federal de Uberlândia; 2006.

15. Rubenstein LZ. Falls in older people: epidemiology, risk factors and strategies for prevention. Age Ageing 2006; 35(Supl. 2):ii37-ii41.

16. National Council on Aging (NCOA) Center for healthy aging model health programs for communities (2007). Washington, DC, Center for Health Aging. [acessado 2013 maio, 31]. Disponível em: http://www.ncoa.org/ improve-health/center-for-healthy-aging/falls-prevention/ 
17. Terra NL. Prevenção de Quedas em Idosos. In: IV Encontro Estadual de Saúde da Pessoa Idosa; 2011 set. 27; Porto Alegre. Porto Alegre: Departamento de Ações em Saúde, Seção da Saúde do Idoso da Secretaria Estadual da Saúde; 2011.

18. Perracini MR, Ramos LR. Fatores associados a quedas em uma coorte de idosos residentes na comunidade. Rev Saude Publica 2002; 36(6):709-716.

19. Siega JC. Quedas na terceira idade. J Bras Med 2007: 92(5):18-22.

20. Mattox D, Feliciano D, Moore E, editores. Trauma. $4^{\text {a }}$ Ed. Porto Alegre: Artmed; 2006.

21. Bulajic-Kopjar M. Seasonal variations in incidence of fracturas among elderly people. Inj Prev 2000; 6(1):1619.

22. Parreira JG, Vianna AMF, Cardoso, GS, Karakhanian WZ, Callil D, Perlingeiro JAG, Soldá SC, Assef JC. Lesões Graves em Vítimas de Queda da Própria Altura. São Paulo: Faculdade de Ciências Médicas da Santa Casa de São Paulo; 2010.

23. Lopes MCL, Violin MR, Lavagnoli AP, Marcon SS. Fatores desencadeantes de quedas no domicílio em uma comunidade de idosos. Coggitare Enferm 2007; 12(4):472-477.

24. Costa AGS, Souza RC, Vitor AF, Araujo TL. Acidentes por quedas em um grupo específico de idosos. Rev. Eletr. Enf. [periódico online]. [acessado 2013 abril, 20]; 13(3):395-404. Disponível em: http://www.fen.ufg.br/ revista/v13/n3/v13n3a04.htm

25. Costa JNA, Gonçalves CDU, Rodrigues GBA, Paula AP, Pereira MM, Saffons MP. Exercícios multisensoriais no equilíbrio. Revista Digital, Buenos Aires [periódico online]. 2009 ago [acessado 2011 nov 25];14:135. Disponível em: http://www.efdeportes.com/efd135/exercicios-multisensoriais-em-idosos. htm

26. Brasil. Ministério da Saúde. Portaria no 2.048/GM, de 5 de novembro de 2002. Regulamenta tecnicamente as urgências e emergências no Sistema Único de Saúde. Diário Oficial da União 2002; 6 nov.

Artigo apresentado em 26/08/2014

Aprovado em 15/04/2015

Versão final apresentada em 17/04/2015 